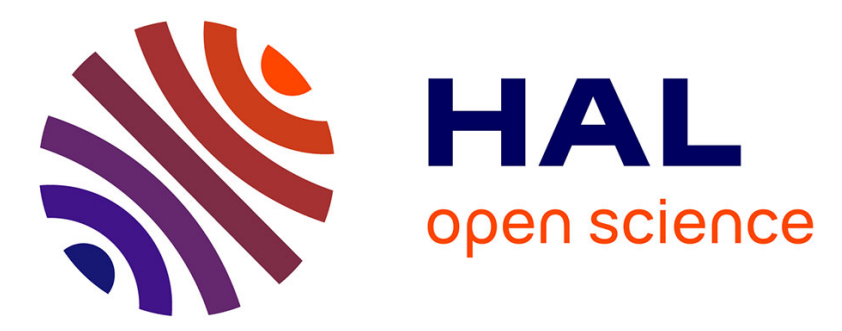

\title{
Estimating maximum and minimum delays for wireless discrete networked control systems
}

\author{
Gilbert Habib, Thierry Divoux, Jean-François Pétin
}

\section{To cite this version:}

Gilbert Habib, Thierry Divoux, Jean-François Pétin. Estimating maximum and minimum delays for wireless discrete networked control systems. Wireless Telecommunications Symposium, WTS 2009, Apr 2009, Prague, Czech Republic. pp.16844746, 10.1109/WTS.2009.5068966 . hal-00417699v2

\section{HAL Id: hal-00417699 \\ https://hal.science/hal-00417699v2}

Submitted on 20 Nov 2009

HAL is a multi-disciplinary open access archive for the deposit and dissemination of scientific research documents, whether they are published or not. The documents may come from teaching and research institutions in France or abroad, or from public or private research centers.
L'archive ouverte pluridisciplinaire HAL, est destinée au dépôt et à la diffusion de documents scientifiques de niveau recherche, publiés ou non, émanant des établissements d'enseignement et de recherche français ou étrangers, des laboratoires publics ou privés. 


\title{
Estimating Maximum and Minimum Delays for Wireless Discrete Networked Control Systems
}

\author{
Gilbert Habib, Thierry Divoux and Jean-François Pétin \\ Centre de Recherche en Automatique de Nancy (CRAN) \\ Nancy-University, CNRS, BP 239, 54506 Vandœuvre-lès-Nancy, France \\ (e-mail:\{Gilbert.Habib, Thierry.Divoux, Jean-Francois.Petin\}@cran.uhp-nancy.fr)
}

\begin{abstract}
The automation architecture consists of actuators, sensors, programmable logic controllers (PLC) and monitoring systems. Some research focuses on how to replace the communication between those entities from wired to wireless communication taking in consideration the industrial constraints, given the advantages of this kind of communication. To study the performance of the Wireless Network System, this paper presents an approach for estimating the maximum and minimum delays for each traffic of a wireless network in the worst case. Influence of the Maximum number of retransmission parameter is studied on the estimated delay and on the packet loss rate. Our results are compared and analyzed with simulated ones obtained with the tool OPNET.
\end{abstract}

\section{INTRODUCTION}

Wireless communication is the revolution method in the factory networks to replace traditional fieldbuses as ProfiBusDP or WorldFip. Given the high benefits like mobility and getting rid from the need for cables, this kind of communication become more useful, but in another way, it presents many weak points that can affect the automation system.

Three main approaches are considered to evaluate the performance of the Network Control System (NCS). The first approach [2], [8], automatic approach, consists in modeling the behavior of the system using timed event graphs and evaluates the response time in Ethernet based automation systems. The second approach [3], network approach, aims to study the performance of a network (Ethernet) to support real communication by calculated the maximum delay (Worst case) using Network Calculus. Finally, the third approach consists on studying the two approaches in the same time, it is called the co-design approach.

Our work consists in estimating the maximum and minimum delays in the worst case in wireless communication taking in consideration noise factor which is an important element in industrial network. These estimation can be used in many ways, off-line by designing control strategies that take into account network performance or on-line by adapt the parameters of the controllers. In our study, we work specifically on the 802.11e technology which offers a level of priority for each traffic.

Section 2 clarifies in details the main problems in industrial systems. 802.11e technology is described in section 3. It is followed by a discussion of related work in section 4 .

Our algorithm to estimate the maximum and minimum delays is presented in section 5. Then, it is applied in different scenarios in section 6. We use OPNET v14.0 to simulate these scenarios in section 7 . Finally in section 8 , a comparison between the estimated and simulated results.

\section{Problematic}

The automation architecture is composed of actuators, sensors, programmable logic controllers (PLC), monitoring systems and finally the network which provide the communication between these entities. This control architecture can be divided into two mains parts: The network part which concern all the communication equipments and the controllers part which concern all the equipment that send or receive data using the network. The communication between the automation equipment must respect many constraints:

1) Real-time communication. In the industrial systems, there exist two kinds of traffic: the acyclic and the cyclic traffic. The acyclic traffic can be an event like an alarm sent by a sensor to the controller system. The cyclic traffic usually is the commands of the controllers to the actuators so that the controllers send periodically packet data to the actuators. In both cases, those traffics must arrive to the destination in bounded delay. In cyclic traffic, the packet data must be delivered to the actuators before the controller begins to send the next packets so the delay must be less than the period of this traffic.

2) Minimum loss packet rate. In the automation systems, the noise rate is an important factor that increases the loss of packets. The data waves propagation may be affected by interference, obstacles, radiation caused by the factory equipments.

Consequently, the communication technology used, must take in consideration all these constraints. We use in our work the wireless communication $802.11 \mathrm{e}$ because of the advantages it offers like mobility and getting rid from the need for cables to communicate so less price cost. This technology suffers from many disadvantages like influence of the loss packet rate by the environment, the non-deterministic medium access control. So the main problem is how to use the 802.11e technology to give a maximum performance in terms of delay, loss rate,...

\section{IEEE $802.11 \mathrm{E}$}

The 802.11e standard provides two Medium Access Control (Mac) mechanisms: the mandatory EDCA (Enhanced Distributed Channel Access) and the optional HCCA (HCF controlled channel access (HCCA)). HCCA provides polled access 
to the medium, QoS AP (Quality Access Point) controls all the traffic that contends the medium. Before that stage, every working station must send a request (ADDTS) to access the medium to the QAP. This request contains traffic information (Maximum MSDU, Minimum Data Rate,...), QAP will then reply by accepting or refusing the demand. EDCA uses the CSMA/CA protocol with priorities to mediate the access to the shared medium. The various streams in stations are classified into eight priorities, referred as User Priorities (UPs) as shown in Table I.

TABLE I

ACCESS CATEGORY MAPPING

\begin{tabular}{|c|c|c|}
\hline $\begin{array}{c}\text { User Priority } \\
(802.1 \mathrm{D})\end{array}$ & Traffic Type & $\begin{array}{c}\text { Access Category } \\
\text { (AC) }\end{array}$ \\
\hline 1 & Background & AC0 \\
\hline 2 & - & AC0 \\
\hline 0 & Best Effort & AC 1 \\
\hline 3 & Excellent Effort & AC1 \\
\hline 4 & Controlled Load & AC2 \\
\hline 5 & Video & AC2 \\
\hline 6 & Voice & AC3 \\
\hline 7 & Network Control & AC3 \\
\hline
\end{tabular}

The UPs are further mapped into four (0-3) Access Categories (AC), AC3 and AC2 represent the voice and video streams that require a delay $<10 \mathrm{~ms}$ and $<100 \mathrm{~ms}$ respectively with minimal jitter; AC1 characterizes the Best effort traffic, and finally $\mathrm{AC} 0$ represents the background traffic.

Each AC has its own queue (FIFO) and is specified by four variables : Access Category Inter-Frame Spaces (AIFS), Transmission Opportunity (TXOP) Limits and Contention Windows (CW(AC)min and $\mathrm{CW}(\mathrm{AC}) \mathrm{max}$ ). If the medium is busy the $\mathrm{AC}$ cannot send packets and must wait until the medium becomes idle for at least AIFS(AC) or EIFS - DIFS + AIFS(AC), before it takes a waiting time called backoffTime calculated as follow:

$$
\text { backoffTime }=\text { backoffNumber } * \text { slot_time }
$$

where,

backoffNumber $=$ integer $(\operatorname{rand}[0, C W(A C)])$, rand [ ] is an uniform distributed function,

slot_time depends on the PHY layer, and $C W(A C)$ min $\leq C W(A C) \leq C W(A C) \max$.

For each packet failure, $\mathrm{CW}(\mathrm{AC})$ is doubled until it reaches $\mathrm{CW}(\mathrm{AC}) \max$, and for each successful packet $\mathrm{CW}(\mathrm{AC})$ shall reset to $\mathrm{CW}(\mathrm{AC}) \mathrm{min}$. The station begins to decrement the backoffTime as long as the medium is idle, and it will send packets when the backoffTime reaches zero. Finally, TXOP limit is the maximal duration for which a station can use the medium for transmission.

Each AC in a station uses the CSMA/CA protocol as if it were alone in the station so that the internal collision or Virtual collision phenomenon may be happen when the backoffTime of ACs in the same station elapses, in this case, AC with higher priority access to the medium and the other defer and try later as it is a real collision. In the EDCA mode, some traffics may also need an admission control to access to the medium so that it maintains two variables admitted_time and used_time. Like in HCCA, each AC in a QSTA transmits a request containing a traffic specification (TSPEC), so when the QAP receives this request and if it accepted, it will send a response to the QSTA containing a variable called medium_time, which represents the amount of time for this traffic to access the medium. While receiving the response, QSTA updates his admitted_time variable to medium_time, used_time is used to count the time that this traffic accessed the medium, it shall not surpass the admitted_time.

\section{RELATED WORK}

To adapt the 802.11e to the control system and to give a better performance, the solution must undergo two mains things. First, it must be accepted by the standard 802.11e, so if any out of norm, it is considered as obsolete solution. Second, the solution can be implemented in the wireless equipment that use the 802.11 e. For example, none of the wireless equipment that exist till now use the HCCA so all the algorithms in development that support the HCCA are considered currently theoretical and cannot be applicable. Many algorithms have been proposed to adapt scheduling for HCCA. In [4], it requires many application parameters like maximum burst size, peak data rate to give the traffic a TXOP to access the medium. In EDCA standard [1], the TXOP limit is static. In the ETXOP [6], the TXOP takes a dynamic value that depends on many elements like priority of the traffic, data rate,... In AEDCF algorithm [9], the author suggests that for every successful transmission, the $\mathrm{CW}$ takes a value depending on the average collision rate and the unsuccessful transmission instead of doubling the old $\mathrm{CW}$, it will be multiplied by a number depending on the traffic priority. In M-EDCF [7], they propose that the backoff value will not freeze when the medium becomes busy but will take a new value depends on the average collision rate. A procedure is presented in [10], where the QAP decides whether the traffic will access in the EDCA or HCCA mode or non of them, by using the requirements (maximum delay and throughput) needed by the traffic. QAP estimates the average delay and throughput that the network can insure to the entry traffic, if there are less than the demand requirements so it will be accepted. Although, in the industrial domain, we are required to work on the maximum estimated delay instead of average delay so that we can abide by the period of the cyclic traffic. Actually, all those algorithms are out of standard or cannot be implemented in the wireless equipment. Our study is accepted by the standard. It consists on estimating the maximum and minimum delays by using the EDCA mode that it is implemented in the wireless devices.

\section{A proposal to COMpUte the Maximum And MINIMUM DELAYS}

The delay, is first dependent on the PHY layer characteristics. OFDM defined by 802.11 a which supports eight different data rates 6, 9, 12, 18, 24, 36, 48 and 54 Mbps is used. A PPDU is formed by PLCP préambule, PLCP header, MPDU, 
tail bits, and pad bits. The PLCP préamble is used for synchronization. The PLCP header contains two fields: the signal field ( 24 bits) that contains information about the rate and length and the service field SRV (16 bits). The PPDU tail bit (6 bits) which are used to return the convolutional encoder to the zero state and finally pad bits are required to make the data bits multiple of OFDM symbols.

So according to [1] and [5], the time to send a packet with size $x$ and in a rate equal to $r$ is calculated as follow:

$$
F(x, r)=T_{s} *\left(5+\operatorname{ceil}\left(\frac{S R V+x+T_{a i l}}{r * T_{s}}\right)\right)
$$

where,

$F(x, r)=$ time to send a packet with size $x$ and in a rate equal to $r$, in $\mu \mathrm{s}$,

$\operatorname{ceil}(A)=$ rounds the element $A$ to the nearest integer greater than or equal to $A$,

$x=$ size of the MPDU,

$r=$ data transmission rate,

$T_{s}($ symbol interval $)=4 \mu \mathrm{s}$,

$S R V=16$ bits,

and $T_{\text {ail }}=6$ bits.

We notice also that the ACK packet is sent in a rate corresponding to the highest mandatory data that is equal to or less than the data transmission rate (r) so they will be understood by all the stations. As previously explained, the EDCA mode uses the CSMA/CA protocol to access the medium, so the minimal delay is estimated when a packet arrives at an empty queue and the medium has been found idle for a time greater than $\operatorname{AIFS}(\mathrm{AC})$. In this case, the station sends immediately the packet. The minimal delay is equal to the time needed to send this packet from the station to the QAP without retransmission. To calculate the maximum delay, every packet in each traffic is studied alone. According to the CSMA/CA protocol, when a packet takes a backoff time, it begins to decrement this value as long as the medium is idle, and it will be frozen when the medium becomes busy (when another stations contend the medium). Consequently, the maximum delay for a packet in study is estimated when it takes a maximum backoffNumber (1), in other words, it equals to $\mathrm{CW}(\mathrm{AC})$, and this value must be frozen the maximum number of times, meaning that other stations that need to access the medium take a backoff that corresponds to this equation:

$(A I F S(A C)+$ backoffNumber)another packet < $(A I F S(A C)+$ backoffNumber) packet studied

and all these stations must have a different $(A I F S(A C)+$ backoffNumber $)$ so that it occupies the medium maximum time. We also suppose that the packets are sent a maximum number of times. Finally, the maximum delay of a traffic is the maximum delay of all his packets. Three main factors may influence the maximum delay:

1) Collision of the packets. In every collision, the sending stations must wait an ACKTimeout before sending another packet. Therefore, the delay will increment. The charge of the network varies this factor, in other words, if we have an important charge, we will have lot of collisions.
2) Noise. As we explained before, in an industrial domain, this factor is important. Packets attacked by the noise are considered as not received by the receiver and this last will not send an ACK, in return the sender will wait an ACKTimeout and resend the packet, and consequently the delay will increment.

3) Maximum number of retransmission (short or long RetryLimit). The sender supposes that the packet is loosed when it resents it a number of times equal to the short or long RetryLimit. The bigger this value, the greater is the number of packets waiting in the queue and consequently, the delay of those packets will increment.

The study will be as follow: Varying the charge of the network and Maximum number of retransmission. In every time, we calculate the maximum delay to all the traffic in the network, if each value is less than its period so this Maximum number of retransmission will be accepted. If not, it will be ignored.

\section{ESTIMATING MAXIMUM AND MINIMUM DELAY}

We work on infrastructure network, it consists of one AP and three stations. Every station sends a cyclic traffic with priority 0,1 or 3 respectively. The data rate in the network is $6 \mathrm{Mb} / \mathrm{s}$. The charge of the network is varied $120,60,30$, $15 \%$ and in each case, Maximum number of retransmission is varied to $2,3,4,5$ or 6 for each traffic. On a $15 \%$ charge, the period of the traffic with priorities 3, 1, 0 are 60, 40, $30 \mathrm{~ms}$ respectively. To vary the charge of the network and to keep the same proportional bandwidth between the traffics, we divide the period of each traffic of the charge before by 2 , example: To obtain a $30 \%$ charge, the period of the traffic with priorities 3,1 and 0 are $30(=60 / 2), 20$ and $15 \mathrm{~ms}$ respectively. We follow the same principle to obtain the periods of the $60 \%$ and $120 \%$ charge. Figure 1 represents the maximum and minimum delay of the traffic with priority equal to 1 on $15 \%$. As we explained before the minimum delay of the traffic is equal to the time needed to send this packet from the station to the QAP without retransmission, so it is independent from the Maximum number of retransmission so that it will be represented as constant horizontal line. In another way, maximum delay increments linearly when we increase the Maximum number of retransmission until it surpass the period of his traffic, in this case, the maximum delay will increment exponentially cause of that the retransmission increments the time waiting for each packet in the queue.

\section{Simulation DELAY AND PACKET LOSS RATE}

Considering the same network (figure 2), we start to vary the charge and the Maximum number of retransmission as before. The noise rate is taken in consideration to increment packet losses. Note that the noise attacks the packet transmission in a manner that will not influence to the CSMA/CA protocol. This rate takes the following values $0,30,50$ and $70 \%$. To simulate this network, we use OPNET v 14.0. Before studying the maximum delay, it is interesting to see the influence of the Maximum number of retransmission on the packet loss rate. Figures 3- 6 represent the packet loss rate 


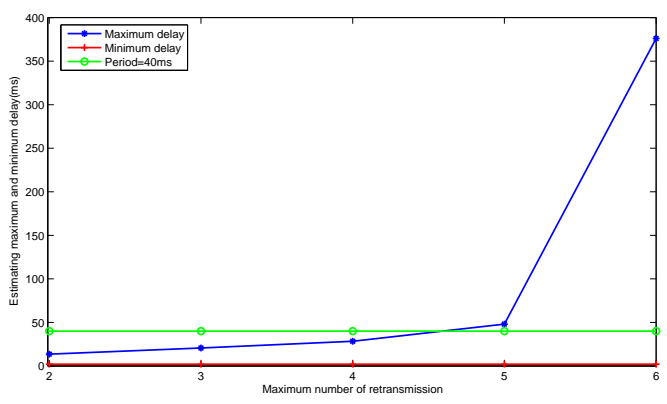

Fig. 1. Estimated Maximum and Minimum delays on charge 15\% for the traffic with priority equal to 1

in function of the Maximum number of retransmission and the noise rate for each charge. On a 15\% charge (figure 3), incrementing the Maximum number of retransmission value decrements the packet loss rate, because sending the packets many times decrement the packet loss rate. Notice that on charge $30 \%$ (figure 4), this phenomenon also exists till Maximum number of retransmission becomes equal to 4 or 5 having the noise rate equal to 70 and $50 \%$ respectively. Afterwards, the packet loss rate will increment, because the retransmission packets may increment the charge of the network so that it will increase the collision of packets. Consequently, it increases the packet loss rate. Finally, when the charge is 60 and 120 $\%$ (figures 5, 6), it is observed that the packet loss rate will increment when Maximum number of retransmission is increased, it plays a reversible effect on the packet loss rate.

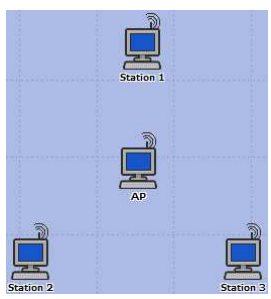

Fig. 2. Simulation Scenario

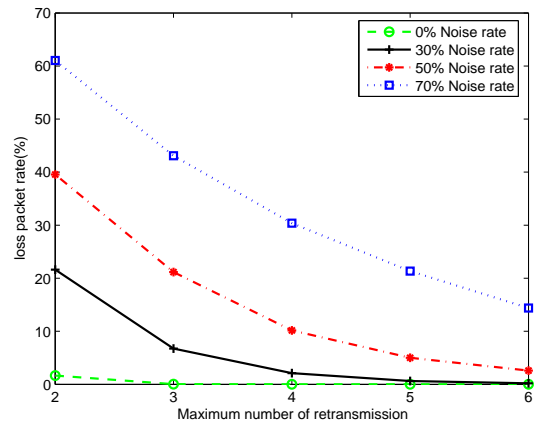

Fig. 3. Packet loss rate on charge $=15 \%$

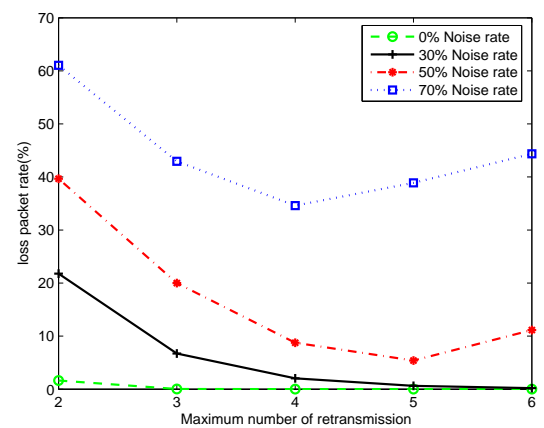

Fig. 4. Packet loss rate on charge $=30 \%$

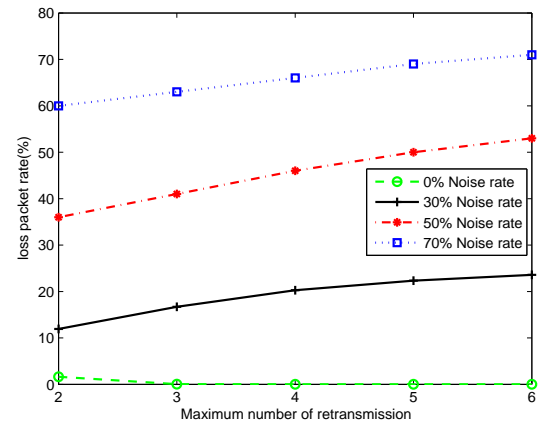

Fig. 5. Packet loss rate on charge $=60 \%$

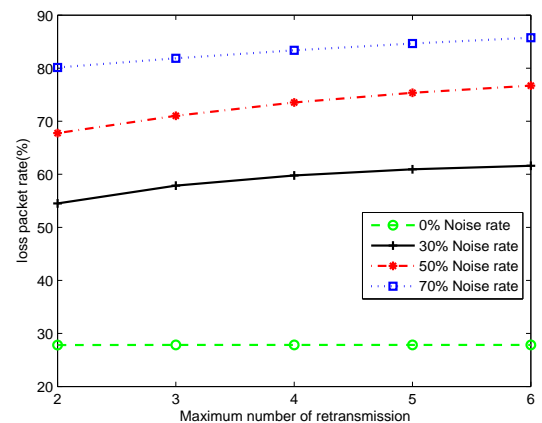

Fig. 6. Packet loss rate on charge $=120 \%$

\section{COMPARISON AND VALIDATION}

Figures 7- 9 represent the estimated maximum delay in every traffic and the simulation maximum delay in different noise rate on a $15 \%$ charge, we can remark that the simulated maximum delay will never exceed the estimated maximum delay. This will valid our work. We can also notice that the difference between simulated and estimated delay increment in function of Maximum number of retransmission cause of that the backoffNumber (1) choose in every retransmission is maximum in the estimated network while in the simulated is random. As we explained before the maximum delay must not surpass its period of his traffic. So in (figure 8), when Maximum number of retransmission is equal to 5, the maximum delay exceeds its period $(40 \mathrm{~ms})$ which makes it unacceptable. If Maximum number of retransmission is equal to 4 , the maximum delay to all the traffics will not surpass its period. In other words, when the charge is equal to $15 \%$ and the Maximum number of retransmission is equal to 4 , the maximum delay of each traffic will not exceed its period. On a 
$30 \%$ charge and when the Maximum number of retransmission is only equal to 2, the maximum delay will not surpass the period of all traffics. Finally on a $60-120 \%$ charge, the maximum delay may always exceeds the period even when the Maximum number of retransmission is minimum.

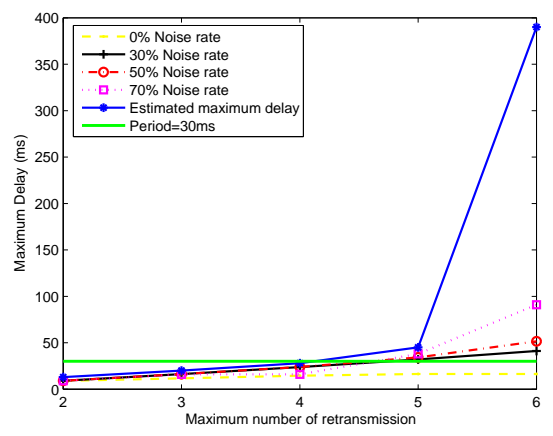

Fig. 7. Estimated/Simulated Maximum delay on charge $=15 \%, P=30 \mathrm{~ms}$

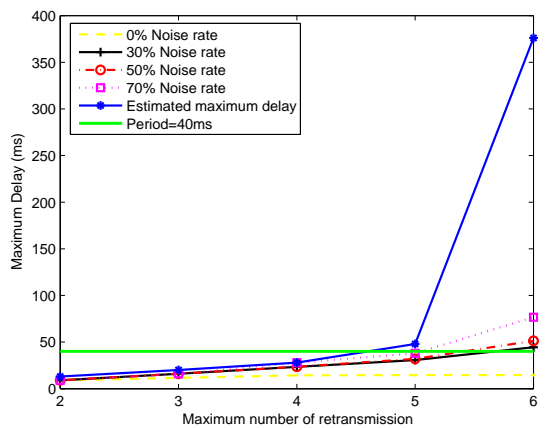

Fig. 8. Estimated/Simulated Maximum delay on charge $=15 \%, P=40 \mathrm{~ms}$

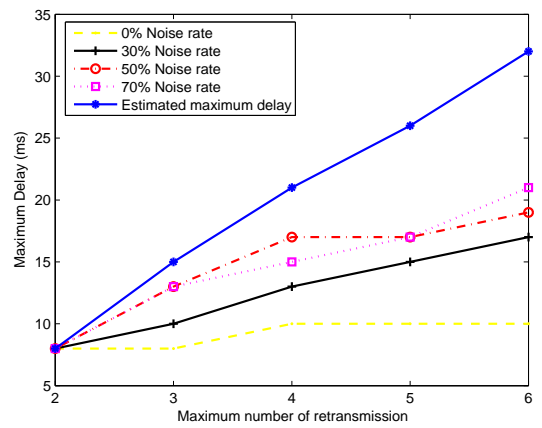

Fig. 9. Estimated/Simulated Maximum delay on charge $=15 \%, P=60 \mathrm{~ms}$

\section{CONCLUSION}

In this paper, we are working in the industrial domain so we are forced to comply by various constraints, so that the maximum delay must not exceed the period of each cyclic traffic. First, the maximum and minimum delay of each traffic in a network using 802.11e are calculated. Second, the Maximum number of retransmission needed that abide by the industrial constraints is estimated. Finally, the calculated and simulated results are compared.

\section{REFERENCES}

[1] IEEE 802.11 WG, Reference number ISO/IEC 8802 11:1999(E) IEEE Std 802.11, 1999 edition. International
Standard [for] Information Technology- Telecommunication and exchange between systems-Local and metropolitan area networks-Specific Requirements - Part11: 'Wireless LAN Medium Access Control (MAC) and Physical Layer (PHY) Specifications”. 1999.

[2] B. Addad and S. Amari. Modeling and Response Time Evaluation of Ethernet-based control Architectures using Timed Event Graphs and Max-Plus Algebra. 4th annual IEEE Conference on Automation Science and Engineering, Washington, USA, 2008.

[3] J. Georges, T. Divoux, and E. Rondeau. Confronting the performances of a switched Ethernet network with industrial constraints by using the network calculus. International journal of communication systems, 18:877903, 18 July 2005.

[4] A. Grilo, M. Macedo, and M. Nunes. A Scheduling Algorithm for QoS support in IEEE 802.11e Networks. IEEE Wireless Communications, 10:36- 43, June 2003.

[5] N. Krommenacker and V. Lecuire. Building industrial communication systems based on IEEE $802.11 \mathrm{~g}$ wireless technology. ETFA'05: 10th IEEE Conference on Emerging Technologies and Factory Automation, Catania, Italy, 19-22 Sept 2005.

[6] A. Ksentini, A. Nafaad, A. Guerouic, and M. Naimib. ETXOP: A resource allocation protocol for QoS-sensitive services provisioning in 802.11 networks. Performance Evaluation, 64:419-443, 2006.

[7] W. Lin and J. Wu. Modified EDCF to improve the performance of IEEE 802.11e WLAN. Computer Communications, 30:841-848, 2007.

[8] P. Meunier, B. Denis, and J. Lesage. Temporal performance evaluation of control architecture in automation systems. EUROSIM'07: Federation of European Simulation Societies, Ljubljana, Slovenia, 9-13 Sept. 2007.

[9] L. Romdhan, Q. Ni, and T. Turletti. Adaptive EDCF: Enhanced Service Differentiation for IEEE 802.11 Wireless Ad-Hoc Networks. WCNC03: IEEE Wireless Communications and Networking Conference, New Orleans, Louisiana, USA, 19-20 March 2003.

[10] R. Zhu, J. Wanga, and M. Ma. Intelligent MAC model for traffic scheduling in IEEE 802.11e wireless LANs. Applied Mathematics and Computation (2008), doi:10.1016/j.amc.2008.05.052. 